\title{
Value of Low-Dose Computed Tomography for Examination after Extracorporeal Shock Wave Lithotripsy for Urolithiasis
}

\author{
Takashi Hatano ${ }^{1 *}$, Kentaro Chikaraishi ${ }^{1}$, Hiroyuki Inaba ${ }^{1}$, Katsuhisa Endo ${ }^{1}$, Mayumi Tamari ${ }^{2}$ \\ and Shin Egawa ${ }^{3}$ \\ ${ }^{1}$ Department of Urology, JR Tokyo General Hospital, Tokyo, Japan \\ ${ }^{2}$ Research Center for Medical Science Core Research Facilities for Basic Science, Jikei University School of Medicine, \\ Tokyo, Japan \\ ${ }^{3}$ Department of Urology, Jikei University School of Medicine, Tokyo, Japan
}

Received: January 3, 2017; Accepted: April 4, 2017; Published: April 15, 2017

*Corresponding author: Takashi Hatano, M.D., Ph.D. Department of Urology, JR Tokyo General Hospital, 2-1-3 Yoyogi Shibuya-ku, Tokyo, 1518528, Japan, Tel: +81-3-3320-2200; Fax: +81-3-3370-8501; E-mail: hatano-t@jreast.co.jp

\begin{abstract}
Objectives: To evaluate the usefulness of low-dose computed tomography after Extracorporeal Shock Wave Lithotripsy (ESWL) for urolithiasis.

Methods: We investigated 103 subjects with urolithiasis who were treated with ESWL. In each case, preoperative plain abdominal films of the Kidney, Ureter and Bladder (KUB) and Standard Computed Tomography (SDCT) and postoperative KUB and Low-Dose Computed Tomography (LDCT) were performed. We compared the Dose Length Product (DLP) and Effective Dose (ED) of SDCT and LDCT. We evaluated any residual calculus by performing postoperative KUB and LDCT, calculating their respective rates of complete fracture, and comparing the performance of both examinations.

Results: The mean DLP and ED were $409 \mathrm{mGy}$ and $6.1 \mathrm{mSv}$ with SDCT, and $103 \mathrm{mGy}$ and $1.5 \mathrm{mSv}$ with LDCT; the dose reduction rate was nearly $75 \%$ in both DLP and ED ( $<<0.001)$. The mean ED of KUB was $1.3 \mathrm{mSv}$, which was similar in dose to LDCT. The accuracy of LDCT was $100 \%$, with no false positives or false negatives. On the other hand, the positive predictive rate of KUB was $90.4 \%$, whereas the negative predictive rate was $75.6 \%$, and the accuracy was $78.6 \%$ $(\mathrm{P}<0.001)$.
\end{abstract}

Conclusion: The exposure dose of LDCT was reduced by $75 \%$ compared with that of SDCT. Our findings suggest that LDCT is likely to be a useful method to assess urinary calculi following ESWL.

Key words: Dose Length Product; Effective Dose; Extracorporeal Shock Wave Lithotripsy; Low-Dose Computed Tomography; Urolithiasis

\section{Abbreviations and Acronyms}

BMI = Body Mass Index; CT = Computed Tomography; Ctdivol $=$ Volume Computed Tomography Dose Index; DLP = Dose Length Product; ED = Effective Dose; ESWL = Extracorporeal Shock Wave Lithotripsy; KUB = Plain Abdominal Films of the Kidney, Ureter and Bladder; LDCT $=$ Low-Dose Computed Tomography; NI = Noise Index; SDCT = Standard Computed Tomography; US = Ultrasound

\section{Introduction}

After ESWL for urolithiasis, KUB, US and CT are widely used for the evaluation of residual calculi [1]. KUB is a conventional imaging technique, but several conditions, such as bowel gas and overlapping of fragments and pelvic bones, often decrease the sensitivity [2]. In addition, KUB cannot evaluate the degree of hydronephrosis. US is effective for assessment of nephrolithiasis and hydronephrosis [3]. However, it is not appropriate for evaluation of the localization or size of ureteral lithiasis. In addition, the diagnostic accuracy of US images is remarkably reduced in obese patients. CT is a well-established technique for the study of the improvement of hydronephrosis and small calculi, but it cannot be performed frequently because of the high doses of radiation exposure $[4,5]$.

LDCT is a method that has been developed to reduce the exposure dose associated with examination, and is mainly performed for lung cancer screening [6,7]. Recently, the benefits of LDCT during the diagnosis and follow-up of urolithiasis have been suggested [8].To improve our understanding the benefits of LDCT scanning for urolithiasis after ESWL, we performed LDCT and assessed the exposure dose and usefulness for examination after ESWL. 


\section{Methods}

Patients and study design: We investigated 103 cases in which ESWL was performed for urolithiasis in our hospital. In each case, preoperative KUB and SDCT and postoperative KUB and LDCT were performed, and exposure dose measurements and treatment effects were evaluated. For treatment effects, we defined a complete fracture as one in which the spall fragments of the treated calculus were less than $3 \mathrm{~mm}$ in size, others being incomplete fractures [9]. We evaluated any residual calculi by performing postoperative KUB and LDCT, calculating their respective rates of complete fracture, and comparing the accuracy of both examinations. In LDCT, evaluation was also performed to determine whether there was any improvement in urinary tract obstruction after treatment. We conducted SDCT once every other follow-up examination to verify the diagnostic accuracy of LDCT and KUB. Radiolucent calculi or the patient with BMI more than 30 were excluded. This study was approved by the institutional review board of JR Tokyo General Hospital (No. H26-10).

Imaging conditions: The CT apparatus used was an Optima CT660 (GE Healthcare, Tokyo, Japan). Imaging conditions were: clear evaluation of the size, localization and configuration of calculi was possible, and diagnosis was possible for the surrounding organs. Settings of the CT were as follows: scanning time: 0.7 seconds/rotation; tube voltage: $120 \mathrm{KV}$; tube current: $50 \mathrm{~mA}$; slice thickness: $5 \mathrm{~mm}$; reconstruction clearance: $5 \mathrm{~mm}$. All images were independently judged by one radiological diagnosis specialist and one urologist.

Dose settings: The dose at the time of LDCT imaging was configured to take into account noise and signal dispersion in the SDCT images. The Noise Index (NI) of SDCT was configured as 8-10, while that of LDCT was configured at around 25. For this reason, the Effective Dose (ED) was reduced by approximately $75 \%$ (Figure 1).
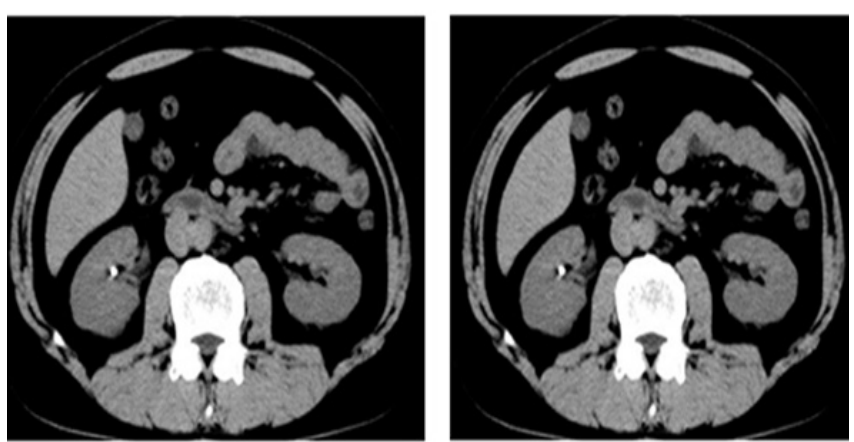

(a)

Figure 1: Comparison of preoperative SDCT and postoperative LDCT

The preoperative SDCT diagnosed a right R2 calculus. The NI was 8.4 and the ED was $6.2 \mathrm{mSv}$ (a). The postoperative LDCT evaluated complete fracture. The NI was 25 and the ED was $1.5 \mathrm{mSv}$ (b). The dose reduction rate of LDCT was $76 \%$.
Detailed settings of the NI were optimized by the radiologist and radiology technician by considering the physical status and localization of the calculi.

Measurement of exposure dose: The exposure dose of the CT examinations was calculated from the dose reports obtained after the examinations. The absorbed dose was measured using a phantom, and defined as CTDIvol, represented in mGy units. The ED was calculated by deriving the Dose Length Product (DLP) using the formula CTDIvol $\times$ L (length of imaging range: $\mathrm{cm}$ ) and multiplying the DLP value by the convention coefficient 0.015 , representing the result in $\mathrm{mSv}$ units.

ESWL: The lithotripter used was the LITHOSTAR Multiline (Siemens, Erlangen, Germany). In each session 3,000 shocks were delivered at a rate of 60 or 90 shock waves per minute. The patients were monitored during the procedure by checking the vital signs heart rate, respiratory rate, blood pressure, and oxygen saturation (pulse oximetry).

\section{Statistical analysis}

All parametric variables were compared by using paired t-tests. All analyses were considered statistically significant with a p-value of $<0.05$.

\section{Results}

\section{Patient characteristics and radiation dose}

\begin{tabular}{|c|c|}
\hline \multicolumn{2}{|c|}{ Table 1: Patient and stone characteristics } \\
\hline \multicolumn{2}{|c|}{ Sex } \\
\hline $\begin{array}{c}\text { Male/Female } \\
\text { Median BMI (kg/m2) } \\
\text { (range) }\end{array}$ \\
\hline \multicolumn{2}{|c|}{ Stone side } \\
\hline Rt/Lt \\
\hline Median stone size (mm) \\
\hline (range) \\
\hline Location \\
\hline R2/R3 \\
\hline U1/U2/U3 \\
\hline
\end{tabular}

Table 1 shows the patient and stone characteristics. Of the 103 patients, 79 were males and 24 were females. Twentyone female patients were of reproductive age. The median stone size was $9 \mathrm{~mm} ; 32$ were renal and 71 were ureteral calculi. The radiation exposure doses with DLP and ED are shown in (Table 2). ). The mean DLP and ED were 409 mGy and $6.1 \mathrm{mSv}$, respectively, with SDCT and $103 \mathrm{mGy}$ and $1.5 \mathrm{mSv}$ with LDCT; the dose reduction rate was nearly $75 \%$ in both DLP and ED (P $<0.001)$. The mean ED of KUB was $1.3 \mathrm{mSv}$ and almost the same dose as with LDCT. 
Table 2: The mean radiation dose according to the CT protocol

\begin{tabular}{c|c|c|c|c|} 
& SDCT & LDCT & $\begin{array}{c}\text { Dose reduction } \\
\text { rate (\%) }\end{array}$ & P-value \\
\hline $\begin{array}{c}\text { DLP } \\
\text { (mGy) }\end{array}$ & $\begin{array}{c}409 \pm \\
137\end{array}$ & $\begin{array}{c}103 \pm \\
36\end{array}$ & 74.8 & $<0.001$ \\
\hline ED (mSv) & $\begin{array}{c}6.1 \pm \\
1.6\end{array}$ & $\begin{array}{c}1.5 \pm \\
0.6\end{array}$ & 75.4 & $<0.001$ \\
\hline
\end{tabular}

Diagnostic performance of postoperative LDCT and KUB

The rate of complete fracture by ESWL was 79.6\% in our study. The diagnostic accuracy of postoperative LDCT and KUB is shown in (Table 3).

Table 3: Diagnostic performance of postoperative LDCT and KUB

\begin{tabular}{|c|c|c|}
\hline Variable & LDCT & KUB \\
\hline True positive & 21 & 19 \\
\hline False positive & 0 & 2 \\
\hline True negative & 82 & 62 \\
\hline False negative & 0 & 20 \\
\hline Positive predictive rate (\%) & 100 & 90.4 \\
\hline Negative predictive rate (\%) & 100 & $75.6^{*}$ \\
\hline Accuracy (\%) & 100 & $78.6^{*}$ \\
\hline *: $\mathrm{P}<0.001$ & & \\
\hline
\end{tabular}

The accuracy of LDCT was $100 \%$, with no false positives or false negatives. On the other hand, for KUB, there were false positives for 2 of 21 cases that were true positives with LDCT, and false negatives for 20 of 82 cases that were true negatives with LDCT. The causes of false negatives included: intestinal gas surrounding the calculus ( 9 cases); overlapping of the calculus and bones due to movement of the calculus ( 7 cases), and others ( 4 cases). The positive predictive rate of KUB was $90.4 \%$, while the negative predictive rate was $75.6 \%$, and the accuracy was $78.6 \%(\mathrm{P}<$ 0.001).

Even if a calculus was clearly found in preoperative KUB, it may not have been sufficiently diagnosed based on postoperative KUB due to intestinal gas and movement of the calculus following surgery (Figure 2). On the other hand, LDCT was able to accurately diagnose the size, shape, and location of the calculus regardless of intestinal gas and movement of the calculus. In addition, LDCT was able to sufficiently evaluate improvement in hydronephrosis and the existence of hematoma. In LDCT, there were no images that were impossible to diagnose due to noise and artifacts in our study.
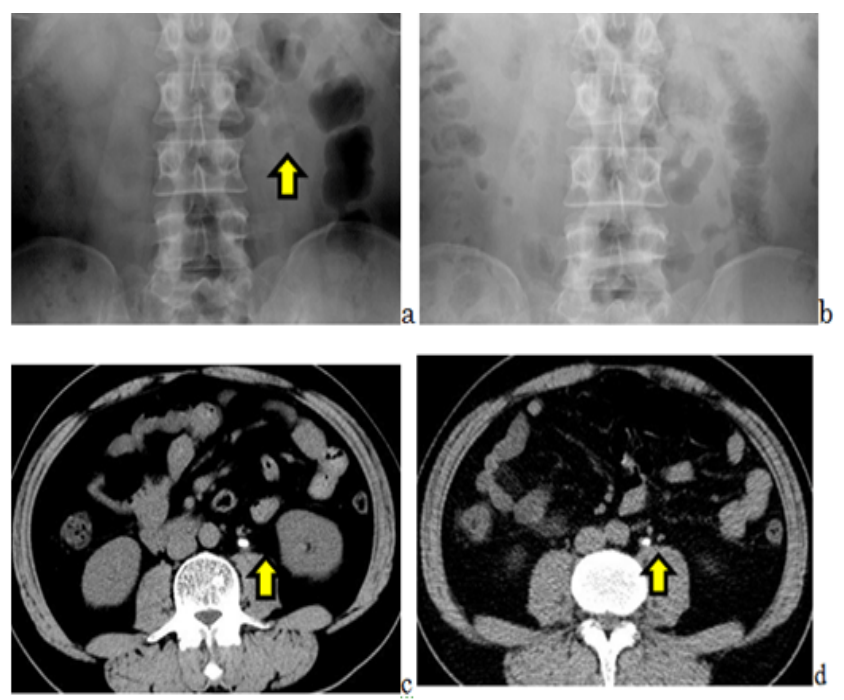

Figure 2: The preoperative KUB and SDCT diagnosed a left U1 calculus $(\mathrm{a}, \mathrm{c})$. The postoperative KUB evaluated complete fracture (b); however, the postoperative LDCT showed a residual calculus (d). KUB was insufficient in this case. The EDs of preoperative SDCT and postoperative LDCT were 7.0 and $2.0 \mathrm{mSv}$, respectively. The dose reduction rate of LDCT was $72 \%$.This patient was obese, so the EDs were higher than the mean dose.

\section{Discussion}

CT provides clearer images and enables more specific diagnostic imaging; however, we should be aware of the radiation risks of CT and keep radiation exposures as low as possible while achieving the required image quality and clinical benefit [10]. Since an earthquake led to a nuclear disaster in Japan in 2011, medical service providers and patients have recently paid more attention to the radiation dose in medical imaging.

Urinary calculi occur in a wide range of age groups from juveniles to the elderly. We must pay careful attention to reproductive cells when conducting diagnostic $\mathrm{X}$ ray examinations. Women aged $12-50$ years are of reproductive age. In this study, $87.5 \%$ of female patients were in this group. In the U.S., $75 \%$ of urinary calculi are assumed to recur and require treatment and follow-up for extended periods $[11,12]$. Accumulative exposure may reach high dosages after X-ray examinations for them. The dosage of radiation exposure during X-ray examinations in Japanese patients is the highest in the world [13]. The majority of exposure is caused by CT. Radical improvement in CT performance has dramatically decreased the exposure dose and enabled the provision of clear images in recent years, and several studies have shown the effectiveness of LDCT for diagnosing urinary calculi [14-16]. ESWL is an operation to shatter the calculus, not to extract it. Therefore, follow-up assessment using medical imaging after the ESWL is very important. We implemented LDCT with the ED reduced by approximately $75 \%$ compared to SDCT. The accuracy of LDCT for urinary calculi after ESWL was $100 \%$. The accuracy of LDCT for urinary calculi greater than 3 $\mathrm{mm}$ in size has also been reported to be $99-100 \%$ in other studies [14]. On the other hand, the accuracy of KUB was $78.6 \%$ in our study. LDCT was also able to accurately diagnose the treatment 
effect and evaluate hydronephrosis and hematoma. The ED of LDCT was around 25\% that of SDCT and equal to that of KUB. As a result, LDCT other than KUB is recommended for examination of urinary calculi following ESWL.

In the U.S., the annual number of CT examinations has increased 20 fold in the last 30 years $[17,18]$. Medical service providers have to pay sufficient attention to the potential risk of second primary cancer in patients due to frequent implementation of CT [19]. The number of CT scanners per million populations in Japan is 2.5 times higher than in the U.S. [17]. Thus, decreasing the exposure dose in CT is a serious matter. The ED of our LDCT was $1.5 \mathrm{mSv}$, approximately equal to KUB. Recently, there have been some reports regarding CT with an extremely low dosage, less than the ED of KUB [20,21]. It is possible to decrease the ED of LDCT by adjusting the NI. However, this decreases the image quality and prevents accurate diagnosis. Diagnosable image quality must be maintained and the dosage should not be blindly decreased in CT. The purpose of inspecting urinary calculi following ESWL is to evaluate the existence, size and location of residual calculi, along with the improvement in hydronephrosis, and the impact on surrounding organs. Optimization of the imaging conditions is vital to obtain sufficient image quality to achieve these examination goals.

There are several limitations of LDCT screening. First, the condition setting of LDCT depends on the model of the CT device. The exposure dose of patients differs even when using the same LDCT due to differences in CT devices [19]. Second, the appropriate value of the ED for urinary calculi cannot be fixed. The ED of our LDCT was $1.5 \mathrm{mSv}$. In prospective diagnoses for urinary calculi, lower dosages may be sufficient for diagnoses by LDCT. We should use SDCT and LDCT in accordance with the purpose of the examination and pay sufficient attention to the exposure of patients to radiation.

In conclusion, LDCT with the exposure dose reduced by $75 \%$ is an effective means of examining the effect of ESWL. For standardization of LDCT, the appropriate setting of the dosage and selection of indications are problems that remain to be resolved. Once this is done, LDCT will be the major means of examining for urinary calculi.

\section{Acknowledgments}

We acknowledge the support and assistance provided by the radiologists and technicians of the Department of Radiology, JR Tokyo General Hospital.

\section{References}

1. Weizer AZ, Auge BK, Silverstein AD, Delvecchio FC, Brizuela RM, Dahm $\mathrm{P}$, et al. Routine postoperative imaging is important after ureteroscopic stone manipulation. J Urol. 2002;168(1):46-50.

2. Levine JA, Neitlich J, Verga M, Dalrymple N, Smith RC. Ureteral calcul in patients with flank pain: correlation of plain radiography with unenhanced helical CT. Radiology. 1997;204(1):27-31. doi: 10.1148/radiology.204.1.9205218

3. Coughlin BF, Risius B, Streem SB, Lorig RJ, Siegel SW. Abdominal radiograph and ultrasound versus excretory urography in the evaluation of asymptomatic patients after extracorporeal shock wave lithotripsy. J
Urol. 1989;142(6):1419-1424.

4. Denton ER, Mackenzie A, Greenwell T, Popert R, Rankin SC. Unenhanced helical CT For renal colic-is the radiation dose justifiable? Clin Radiol. 1999;54(7):444-447.

5. Prakash P, Kalra MK, Kambadakone AK, Pien H, Hsieh J, Blake MA, et al. Reducing abdominal CT radiation dose with adaptive statistical iterative reconstruction technique. Invest Radiol. 2010; 45(4):202-210. doi: 10.1097/RLI.ob013e3181dzfeec

6. The National Lung Screening Trial Research Team. Reduced lung-cancer mortality with low-dose computed tomographic screening. N Engl J Med. 2011;365:395-409. doi: 10.1056/NEJMoa1102873

7. Kobatashi T, Kibe Y, Hiura T, et al. Evaluation of the exposed dose in a low dose CT for lung cancer screening to its permitted image quality. J Thorac CT Screen. 2014;21:30-35.

8. Chen TT, Wang C, Ferrandino MN, Scales CD, Yoshizumi TT, Preminger $\mathrm{GM}$, et al. Radiation exposure during the evaluation and management of nephrolithiasis. J Urol. 2015;194(4):878-885. doi: 10.1016/j. juro.2015.04.118

9. Lopes Neto AC, Korkes F, Silva JL, Amarante RD, Mattos MH, TobiasMachado $\mathrm{M}$, et al. Prospective randomized study of treatment of a large proximal ureteral stoned. J Urol. 2012;187(1):164-168. doi: 10.1016/j.juro.2011.09.054

10. Nickoloff EL, Alderson PO. Radiation exposure to patients from CT: public perception, and policy. Am J Roentgenol. 2001:177(2):285-287.

11.Curhan GC. Epidemiology of stone disease. Urol Clin North Am. 2007;34:287-293. doi: 10.1016/j.ucl.2007.04.003

12. Bartoletti R, Cai T, Mondaini N, Melone F, Travaglini F, Carini M, et al. Epidemiology and risk factors in urolithiasis. Urol Int. 2007;79:3-7. doi: 10.1159/000104434

13. Gonzalez AB, Darby S. Risk of cancer from diagnostic X-rays: estimates for the UK and 14 other countries. Lancet. 2004;363(9406):345-351. doi: 10.1016/S0140-6736(04)15433-0

14. Kwon JK, Chang IH, Moon YT, Lee JB, Park HJ, Park SB. Usefulness of low-dose nonenhanced computed tomography with iterative reconstruction for evaluation of urolithiasis: diagnosis performance and agreement between the urologist and the radiologist. Urology. 2015; 85(3):531-538. doi: 10.1016/j.urology.2014.11.021

15.Sohn W, Clayman RV, Lee JY, Cohen A, Mucksavage P. Low-dose and standard computed tomography scans yield equivalent stone measurements. Urology. 2013;81(2):231-234. doi: 10.1016/j.urology.2012.09.049

16. Park SH, Kim KD, Moon YT, Myung SC, Kim TH, Chang IH, et al. Pilot study of low-dose nonnhanced computed tomography with iterative reconstruction for diagnosis of urinary stones. Korean J Urol. 2014;55(9):581-586. doi: 10.4111/kju.2014.55.9.581

17. Hendee WR, Becker GJ, Borgstede JP, Bosma J, Casarella WJ, Erickson $\mathrm{BA}$, et al. Addressing overutilization in medical imaging. Radiology. 2010;257(1):240-245. doi: 10.1148/radiol.10100063

18. Brenner DJ, Hall EJ. Computed tomography?an increasing source of radiation exposure. N Engl J Med. 2007;357:2277-2284. doi: 10.1056/ NEJMra072149

19. Pandharipande PV, Eisenberg JD, Lee RJ, Gilmore ME, Turan EA, Singh $\mathrm{S}$, et al. Patients with testicular cancer undergoing CT surveillance demonstrate a pitfall of radiation-induced cancer risk estimates: the 
timing paradox. Radiology. 2013;266(3):896-904. doi: 10.1148/radiol.12121015

20.Glazer DI, Maturen KE, Cohan RH, Davenport MS, Ellis JH, Knoepp US, et al. Assessment of $1 \mathrm{mSv}$ urinary tract stone $\mathrm{CT}$ with model-based iterative reconstruction. AJR Am J Roentgenol. 2014;203(6):1230-1235. doi: 10.2214/AJR.13.12271

21. Scott Kriegshauser J, Naidu SG, Paden RG, He M, Wu Q, Hara AK. Feasibility of ultra-low radiation dose reduction for renal stone CT using model-based iterative reconstruction: prospective pilot study. Clin Imaging. 2015;39(1):99-103. doi: 10.1016/j.clinimag.2014.10.013 